\title{
Clinical Manifestations, Treatment, and Outcome of Hospitalized Patients with Plasmodium vivax Malaria in Two Indian States: A Retrospective Study
}

\author{
Jagjit Singh,, Bhargav Purohit, ${ }^{2}$ Anupama Desai, ${ }^{3}$ Lalita Savardekar, \\ Preeti Shanbag, ${ }^{5}$ and Nilima Kshirsagar ${ }^{6}$ \\ ${ }^{1}$ Department of Pharmacology, Government Medical College and Hospital, Chandigarh 160035, India \\ ${ }^{2}$ Department of Pharmacology, GMERS Medical College, Sola, Ahmedabad, Gujarat 382012, India \\ ${ }^{3}$ Department of Pharmacology, Surat Municipal Institute of Medical Sciences \& Research (SMIMER), Surat, Gujarat 395010, India \\ ${ }^{4}$ National Institute of Research in Reproductive Health, Parel, Mumbai 400012, India \\ ${ }^{5}$ ESI-PGIMSR \& MGM Hospital, Parel, Mumbai 400012, India \\ ${ }^{6}$ National Chair Clinical Pharmacology, Indian Council of Medical Research, ESI-PGIMSR \& MGM Hospital, Parel, \\ Mumbai 400012, India
}

Correspondence should be addressed to Preeti Shanbag; pshanbag@gmail.com

Received 30 September 2013; Revised 4 December 2013; Accepted 10 December 2013

Academic Editor: Rana Chattopadhyay

Copyright ( 2013 Jagjit Singh et al. This is an open access article distributed under the Creative Commons Attribution License, which permits unrestricted use, distribution, and reproduction in any medium, provided the original work is properly cited.

This was a retrospective study done on 110 patients hospitalized with $P$. vivax malaria in three medical college hospitals, one in the union territory of Chandigarh and the other two in Gujarat, that is, Ahmedabad and Surat. The clinical presentation, treatment, and outcome were recorded. As per WHO criteria for severity, 19 of 110 patients had severe disease - six patients had clinical jaundice with hepatic dysfunction, three patients had severe anemia, three had spontaneous bleeding, two had acute respiratory distress syndrome, and one had cerebral malaria, hyperparasitemia, renal failure, circulatory collapse, and metabolic acidosis. All patients with severe $P$. vivax malaria survived, but one child with cerebral malaria had neurological sequelae. There was wide variation in the antimalarial treatment received at the three centres. Plasmodium vivax malaria can no longer be considered a benign condition. WHO guidelines for treatment of $P$. vivax malaria need to be reinforced.

\section{Introduction}

India accounts for $77 \%$ of the total malaria in south-east Asia with Plasmodium vivax being responsible for more than $50 \%$ of the cases. Although earlier regarded as causing a benign infection, there is increasing evidence that the overall burden, economic impact, and severity of $P$. vivax have been underestimated [1]. There are only three large studies from India which describe the whole spectrum of severe clinical manifestations of $P$. vivax malaria [2-4].

Also, malaria case management remains a vital component of malaria control strategies. The WHO guidelines for the treatment of malaria recommend chloroquine (except in areas of chloroquine resistance) as first-line treatment for uncomplicated $P$. vivax malaria with 14 days of primaquine for radical cure. The WHO guidelines for the treatment of malaria recommend that intravenous artesunate should be used for the treatment of severe malaria (falciparum and vivax) for a minimum period of 24 hours. Following initial parenteral treatment, once the patient can tolerate oral therapy, it is essential to continue and complete treatment with an effective oral antimalarial using a full course of an effective ACT (artesunate plus amodiaquine or artemether plus lumefantrine or dihydroartemisinin plus piperaquine) or artesunate (plus clindamycin or doxycycline) or quinine (plus clindamycin or doxycycline) [5].

The present study was done to evaluate the clinical manifestations and outcome in patients hospitalized with $P$. vivax malaria and whether WHO guidelines for treatment of $P$. vivax malaria were being adhered to. 


\section{Methods}

2.1. Design and Setting of Study. This was a retrospective study done using data collected from the medical records of three tertiary care centres. One of the hospitals was in Chandigarh, a union territory in the northern region of India, whereas two of the hospitals were in Gujarat (Sola, Ahmedabad and Surat), a state in the western region of India. Records of patients admitted to the ward or intensive care unit of the respective hospitals, from May to December 2012, with a diagnosis of $P$. vivax malaria, were studied. The period from May to December was chosen since it coincides with the monsoons in India, a period when transmission of malaria is at its highest $[6,7]$.

2.2. Target Population. Adults and children, with smearpositive $P$. vivax malaria were included in the study. Patients of $P$. falciparum malaria, those with mixed infections and those with other coexisting infections like typhoid fever, dengue, and leptospirosis, were excluded.

2.3. Tool for Data Collection. A case record form was developed and used to retrieve data from the inpatient records. The first three authors were responsible for extracting data from the medical records of their respective hospitals.

2.4. Ethical Issues. Ethics committee approval was obtained from all three hospitals for retrieval of data from the medical records. The confidentiality of the patients' record and identity of the treating physician was adequately protected using codes.

2.5. Data Extraction. Information extracted from the records included patient's age, sex, the presenting complaints, findings on clinical examination, hematological and biochemical investigations, treatment given, course in the hospital, and outcome.

In the absence of guidelines to classify severe $P$. vivax malaria, the WHO guidelines for classification of severe $P$. falciparum malaria were used to identify patients with severe disease [6].

2.6. Data Management. The data was pooled at the coordinating centre at the National Institute for Research in Reproductive Health, Mumbai. Data entry and analysis were done using Microsoft Excel 2007. Descriptive statistics in terms of proportions for qualitative variables were applied for analysis of data.

\section{Results}

3.1. Patient Characteristics. Medical records of a total of 110 patients hospitalized for $P$. vivax malaria in the three centres were studied. The ages of the patients are summarized in Table 1. Twenty-four of these 110 patients were children less than 12 years of age.

3.2. Clinical Features. The presenting symptoms are summarised in Table 2. Fever was the commonest symptom being
TABLE 1: Age of patients.

\begin{tabular}{lc}
\hline Age in years & Number of patients \\
\hline $0-12$ & 24 \\
$13-20$ & 21 \\
$21-40$ & 43 \\
$41-60$ & 17 \\
$>60$ & 5 \\
\hline Total & 110 \\
\hline
\end{tabular}

TABLE 2: Symptoms of patients hospitalized with $P$. vivax malaria in the three centres.

\begin{tabular}{lc}
\hline Symptom & Number $(n=110)$ \\
\hline Fever & 110 \\
Chills & 86 \\
Headache & 53 \\
Bodyache & 44 \\
Seizures & 03 \\
\hline
\end{tabular}

present in all the patients followed by chills which were present in 86 of 110 patients.

3.3. Severe Disease. Nineteen of 110 patients (17.2\%) hospitalized with $P$. vivax malaria had severe disease (Tables 3 and 4). Jaundice with evidence of other vital organ dysfunction was seen in eight patients. Severe anemia was seen in five patients. Though 38 patients had thrombocytopenia, only three had bleeding manifestations (epistaxis-1, hematemesis, and malena-2).

3.4. Treatment. The treatment and outcome of patients with severe disease is given in Table 5.

The five patients with severe anemia received packed cell transfusions. Three patients with thrombocytopenia (platelet counts of $12,000 / \mathrm{cu} \cdot \mathrm{mm}, 26,000 / \mathrm{cu} \cdot \mathrm{mm}$, and $23,000 / \mathrm{cu} \cdot \mathrm{mm}$ ) had bleeding manifestations and received platelet transfusions. Two patients with ARDS required ventilation. The one patient with acute renal failure required dialysis, with indications being anuria and hyperkalemia.

3.5. Outcome. All patients with $P$. vivax malaria including those with severe disease survived. One child with cerebral malaria had sequelae in the form of blindness which was still present at one-month followup.

\section{Discussion}

Nineteen of 110 (17.2\%) hospitalized patients with P. vivax malaria had evidence of severe disease. Only three large studies describing the whole spectrum of severe manifestations with $P$. vivax malaria could be identified from India; two were from Bikaner by Kochar et al. [2,3], while the third was from Mumbai by Nadkar et al. [4]. Severe disease was seen in $63.1 \%$ of children as compared to $8.8 \%$ of adults hospitalized for $P$. vivax malaria in Bikaner. The criteria for hospitalizing patients with $P$. vivax malaria were not well defined in our 
TABLE 3: Hematological parameters of patients with severe vivax malaria.

\begin{tabular}{|c|c|c|c|c|c|c|c|c|}
\hline No. & $\begin{array}{c}\mathrm{Hb} \\
(\mathrm{g} / \mathrm{dL})\end{array}$ & $\begin{array}{c}\text { Total count } \\
10^{3} / \mathrm{mm}^{3} \\
\end{array}$ & $\begin{array}{c}\text { Platelet count } \\
10^{3} / \mathrm{mm}^{3} \\
\end{array}$ & $\begin{array}{c}\text { Blood } \\
\text { sugar mg/dL }\end{array}$ & $\begin{array}{c}\text { Blood urea } \\
\text { nitrogen } \mathrm{mg} / \mathrm{dL}\end{array}$ & $\begin{array}{c}\text { Serum } \\
\text { creatinine } \mathrm{mg} / \mathrm{dL}\end{array}$ & $\begin{array}{c}\text { Serum bilirubin mg/dL } \\
\text { conjugated/unconjugated }\end{array}$ & $\begin{array}{c}\text { Serum } \\
\text { ALT IU/L }\end{array}$ \\
\hline 1 & 8.0 & 5.9 & 90 & 94 & 24 & 0.9 & $1.0 / 0.4$ & 40 \\
\hline 2 & 7.0 & 4.8 & 12 & 90 & 38 & 1.2 & $0.9 / 0.3$ & 30 \\
\hline 3 & 9.5 & 7.6 & 50 & 105 & 94 & 1.6 & $10.3 / 6.5$ & 360 \\
\hline 4 & 4.5 & 9.9 & 121 & 65 & 90 & 1.5 & $3.1 / 1.8$ & 107 \\
\hline 5 & 9.6 & 6.2 & 110 & 120 & 120 & 3.2 & $3.6 / 2.2$ & 97 \\
\hline 6 & 6.8 & 4.8 & 26 & 60 & 60 & 0.8 & $1.0 / 0.6$ & 40 \\
\hline 7 & 6.9 & 8.4 & 87 & 138 & 28 & 0.7 & $1.4 / 0.8$ & 38 \\
\hline 8 & 8.4 & 5.0 & 96 & 90 & 52 & 1.3 & $16.4 / 10.9$ & 520 \\
\hline 9 & 7.2 & 3.7 & 80 & 130 & 30 & 0.8 & $8.4 / 6.4$ & 368 \\
\hline 10 & 9.7 & 4.5 & 96 & 105 & 42 & 1.4 & $4.2 / 3.1$ & 287 \\
\hline 11 & 4.8 & 7.2 & 120 & 110 & 22 & 0.9 & $1.6 / 1.0$ & 89 \\
\hline 12 & 3.8 & 9.1 & 110 & 95 & 19 & 1.0 & $1.4 / 0.9$ & 75 \\
\hline 13 & 8.6 & 9.8 & 23 & 70 & 42 & 1.1 & $6.8 / 4.3$ & 356 \\
\hline 14 & 8.4 & 10.0 & 75 & 95 & 38 & 1.5 & $1.8 / 1.2$ & 98 \\
\hline 15 & 7.8 & 6.8 & 68 & 128 & 36 & 1.4 & $5.6 / 3.9$ & 276 \\
\hline 16 & 9.5 & 5.6 & 15 & 112 & 28 & 1.1 & $1.2 / 0.6$ & 40 \\
\hline 17 & 10.0 & 8.7 & 35 & 96 & 25 & 1.3 & $1.6 / 1.1$ & 52 \\
\hline 18 & 5.0 & 9.6 & 45 & 102 & 35 & 1.4 & $1.7 / 1.2$ & 60 \\
\hline 19 & 4.6 & 10.2 & 20 & 88 & 40 & 1.2 & $1.0 / 0.3$ & 42 \\
\hline
\end{tabular}

TABLE 4: Severe P. vivax malaria.

\begin{tabular}{|c|c|c|c|c|}
\hline & Chandigarh & Sola, Ahmedabad & Surat & Total \\
\hline $\begin{array}{l}\text { Total number hospitalized with } \\
P \text {. vivax malaria }\end{array}$ & 22 & 59 & 29 & 110 \\
\hline $\begin{array}{l}\text { Number with severe } P \text {. vivax } \\
\text { malaria }\end{array}$ & 7 & 6 & 6 & 19 \\
\hline Cerebral malaria & 1 & 2 & 0 & 3 \\
\hline Circulatory collapse & 1 & 0 & 0 & 1 \\
\hline Acute renal failure & 1 & 0 & 0 & 1 \\
\hline Jaundice & 2 & 2 & 4 & 8 \\
\hline $\begin{array}{l}\text { Acute respiratory distress } \\
\text { syndrome }\end{array}$ & 1 & 0 & 1 & 2 \\
\hline Severe anemia & 1 & 2 & 2 & 5 \\
\hline Thrombocytopenia & 7 & 23 & 8 & 38 \\
\hline Hyperparasitemia & 0 & 0 & 1 & 1 \\
\hline Metabolic acidosis & 0 & 0 & 1 & 1 \\
\hline
\end{tabular}

study or in the published studies and were at the discretion of the admitting physician.

Severe anemia (hemoglobin $<5 \mathrm{G} / \mathrm{dL}$ ) necessitating packed red cell transfusion was seen in five of nineteen patients with severe malaria in this study. The low parasite biomass of $P$. vivax indicates that severe anemia is not due to destruction of infected RBCs alone. Malariotherapy studies have shown that, for every infected RBC destroyed during vivax infection, 32 noninfected RBCs are removed from the circulation, compared to the loss of $8 \mathrm{RBCs}$ for every infected erythrocyte in falciparum malaria. Cytokine-related dyserythropoiesis also probably contributes to anemia [8].

Thrombocytopenia was seen in $34.55 \%$ of patients hospitalized with vivax malaria. Bleeding manifestations necessitating platelet transfusions were seen in only three patients. The frequency of thrombocytopenia, that is, platelet count $<1,50,000 / \mathrm{cu} \cdot \mathrm{mm}$, in malaria ranges from $24 \%$ to $94 \%$ in the literature and this frequency is not different in the two species. However, occurrence of bleeding even in severe illness is low and a conservative approach is adopted in most cases. Platelet 
TABLE 5: Treatment and outcome of patients with severe disease.

\begin{tabular}{lccc}
\hline & Chandigarh & Sola & Surat \\
\hline Patients with severe malaria & $7(33.33 \%)$ & $6(10.17 \%)$ & $6(20.08 \%)$ \\
Artesunate alone & 4 & 0 & 1 \\
Chloroquine & 0 & 5 & 5 \\
Artemisinin-based & 3 & 1 & 0 \\
combination therapy (ACT) & & 6 & 5 \\
Primaquine & 0 & 2 & 2 \\
Packed cell transfusion & 1 & 0 & 0 \\
Platelet transfusion & 3 & 0 & 1 \\
Ventilation & 2 & 0 & 0 \\
Dialysis & 1 & 0 & 0 \\
Sequelae & 1 & 0 & 0 \\
Death & 0 & &
\end{tabular}

counts usually revert to normal after effective antimalarial treatment [9].

Cerebral malaria was seen in three of nineteen patients with severe vivax malaria in our study. Cytoadherence phenomena are believed to be central to the etiology of cerebral malaria in falciparum infection, but their role in $P$. vivax malaria remains unclear [10].

One patient each from Chandigarh and Surat had acute respiratory distress syndrome (ARDS) and required ventilatory support. ARDS is considered to be the most severe form of acute lung injury in malaria and has been reported most commonly with $P$. falciparum malaria. Case reports of ARDS with $P$. vivax malaria are also being increasingly reported [11].

Clinical jaundice with hepatic dysfunction was seen in eight of nineteen patients with severe vivax malaria. Hepatic dysfunction has been also described in $20-58 \%$ of patients with vivax malaria in Bikaner and Mumbai [2-4].

The WHO guidelines recommend that severe $P$. vivax malaria be treated along the lines of severe falciparum malaria with artemisinin-based combination therapies (ACT) [5].

There was wide variation in the therapy for severe disease in the three centres. Chloroquine was used as the antimalarial of choice in Sola even in patients with severe disease. In Surat, both artesunate and chloroquine were used in patients with severe $P$. vivax malaria, whereas in Chandigarh artesunate only was used in four patients. ACT was used in four patients (three in Chandigarh and one in Surat). The rationale for using antimalarial combination therapy is the simultaneous use of two or more blood schizontocidal medicines with independent modes of action and, thus, different biochemical targets in the parasite. This is postulated to prevent or delay the appearance of resistance [5].

Drug resistance to the first-line drug chloroquine has been reported from Papua New Guinea, Indonesia, parts of Asia including India, and South America [12-14]. Chloroquine should be used as the drug of first choice in vivax malaria due to the low prevalence of $1-2 \%$ resistance in these patients. This is particularly important for countries like India where between 60 and $70 \%$ of the cases are caused by vivax [1]. However, surveillance for drug resistance is recommended and drug policy should be based on surveillance data.

WHO guidelines recommend primaquine for 14 days for radical cure in $P$. vivax malaria [5]. None of the patients in Chandigarh received primaquine due to temporary nonavailability of kits for estimation of G6PD level. All hospitalized patients with $P$. vivax malaria in Sola and Surat received primaquine in the correct dosage.

There were no deaths in any of the patients hospitalized with $P$. vivax malaria at any of the three centres. One child with cerebral malaria in Chandigarh had neurological sequelae at discharge. A mortality of $6 \%$ was noted in children in Bikaner, whereas it was $5 \%$ and $0.9 \%$ in adults in Bikaner and Mumbai, respectively [2-4].

The limitations of this study are as follows: it is a retrospective study with a small sample size and uses data retrieved from the medical records. The problem of mixed infection has not been addressed adequately since this was a retrospective study and PCR is not done routinely at the three centres. A prospective multicentric study with an additional centre in Mumbai is under way and will no doubt clarify the picture better.

\section{Conclusions}

Plasmodium vivax can result in severe disease and can no longer be considered to be a benign condition in India. There is considerable variation in the treatment of $P$. vivax malaria. Adherence to WHO guidelines for treatment is recommended.

\section{Conflict of Interests}

The authors declare that they have no conflict of interests.

\section{Acknowledgments}

This study was done as part requirement of an "Idea to Manuscript-Outcomes Research Workshop" funded by the Indian Council of Medical Research and held in Mumbai, India, in April 2012. The study was presented as a paper at the Sixth International Annual Conference of the South Asian Chapter of the American College of Clinical Pharmacology held in Mumbai, India, from 21 to 22 April 2013. The authors also thank Plasmodium vivax Malaria Study Group Government Medical College \& Hospital, Chandigarh: Dr. Ram Singh, Dr. C. S. Gautam, and Dr. Rajiv Kumar; GMERS Medical College, Sola, Ahmedabad, Gujarat: Dr. Chetna Desai, Dr. B. D. Mankad, and Dr. Nehal Patel; SMIMER, Surat, Gujarat: Dr. Ashok Chaudhary and Dr. Poonam Singh.

\section{References}

[1] A. Kumar, N. Valecha, T. Jain, and A. P. Dash, "Burden of malaria in India: retrospective and prospective view," The American Journal of Tropical Medicine and Hygiene, vol. 77, no. 6, pp. 69-78, 2007. 
[2] D. K. Kochar, G. S. Tanwar, P. C. Khatri et al., "Clinical features of children hospitalized with malaria-a study from Bikaner, Northwest India," American Journal of Tropical Medicine and Hygiene, vol. 83, no. 5, pp. 981-989, 2010.

[3] D. K. Kochar, A. Das, S. K. Kochar et al., "Severe Plasmodium vivax malaria: a report on serial cases from Bikaner in northwestern India," American Journal of Tropical Medicine and Hygiene, vol. 80, no. 2, pp. 194-198, 2009.

[4] M. Y. Nadkar, A. M. Huchche, R. Singh, and A. R. Pazare, "Clinical profile of severe Plasmodium vivax malaria in a tertiary care centre in Mumbai from June 2010 -January 2011," Journal of the Association of Physicians of India, vol. 60, pp. 1113, 2012.

[5] WHO Guidelines for the Treatment of Malaria, World Health Organization, Geneva, Switzerland, 2nd edition, 2010.

[6] S. K. Sharma, R. Chattopadhyay, K. Chakrabarti et al., "Epidemiology of malaria transmission and development of natural immunity in a malaria-endemic village, San Dulakudar, in Orissa state, India," American Journal of Tropical Medicine and Hygiene, vol. 71, no. 4, pp. 457-465, 2004.

[7] N. Pemola Devi and R. K. Jauhari, "Climatic variables and malaria incidence in Dehradun, Uttaranchal, India," Journal of Vector Borne Diseases, vol. 43, no. 1, pp. 21-28, 2006.

[8] N. M. Anstey, B. Russell, T. W. Yeo, and R. N. Price, "The pathophysiology of vivax malaria," Trends in Parasitology, vol. 25, no. 5, pp. 220-227, 2009.

[9] M. V. G. Lacerda, M. P. G. Mourão, H. C. Coelho, and J. B. Santos, "Thrombocytopenia in malaria: who cares?" Memorias do Instituto Oswaldo Cruz, vol. 106, supplement 1, pp. 52-63, 2011.

[10] C. R. J. C. Newton, T. T. Hien, and N. White, "Cerebral malaria," Journal of Neurology Neurosurgery and Psychiatry, vol. 69, no. 4, pp. 433-441, 2000.

[11] W. R. Taylor, J. Hanson, G. D. Turner, N. J. White, and A. M. Dondorp, "Respiratory manifestations of malaria," Chest, vol. 142, pp. 492-505, 2012.

[12] J. K. Baird, "Chloroquine resistance in Plasmodium vivax," Antimicrobial Agents and Chemotherapy, vol. 48, no. 11, pp. 4075-4083, 2004.

[13] M. Garg, N. Gopinathan, P. Bodhe, and N. A. Kshirsagar, "Vivax malaria resistant to chloroquine: case reports from Bombay," Transactions of the Royal Society of Tropical Medicine and Hygiene, vol. 89, no. 6, pp. 656-657, 1995.

[14] E. J. Phillips, J. S. Keystone, and K. C. Kain, "Failure of combined chloroquine and high-dose primaquine therapy for Plasmodium vivax malaria acquired in Guyana, South America," Clinical Infectious Diseases, vol. 23, no. 5, pp. 1171-1175, 1996. 


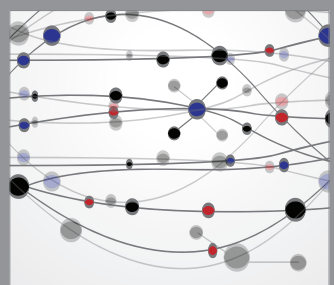

The Scientific World Journal
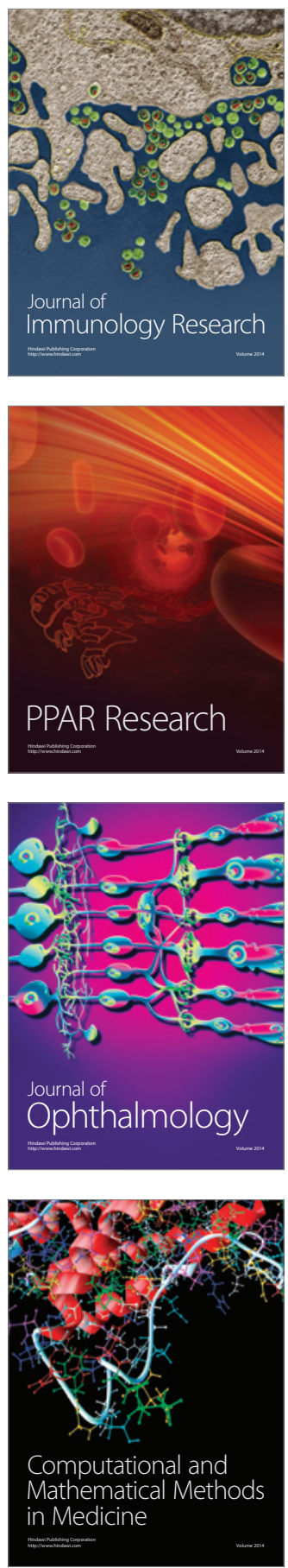

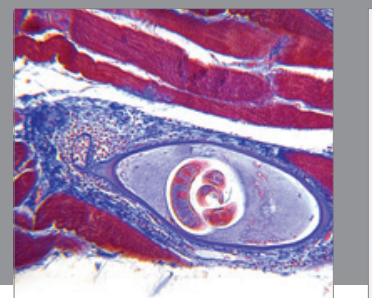

Gastroenterology

Research and Practice
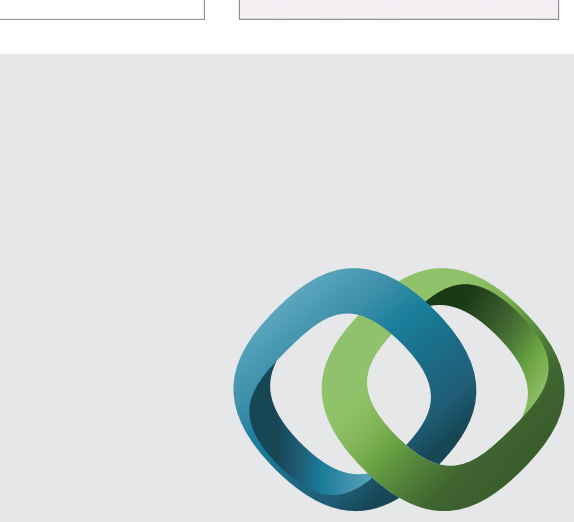

\section{Hindawi}

Submit your manuscripts at

http://www.hindawi.com
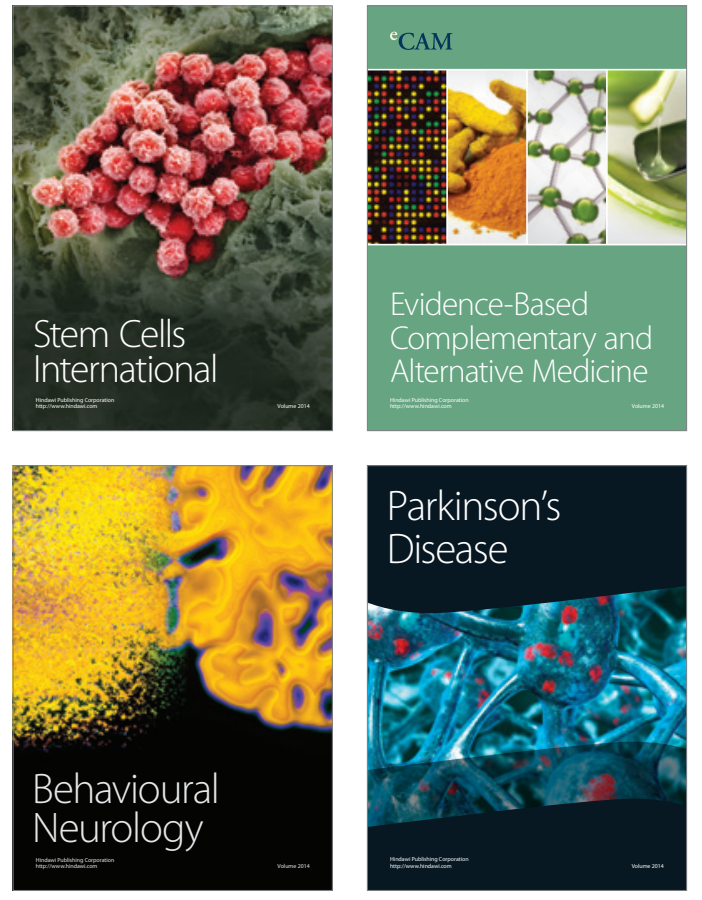
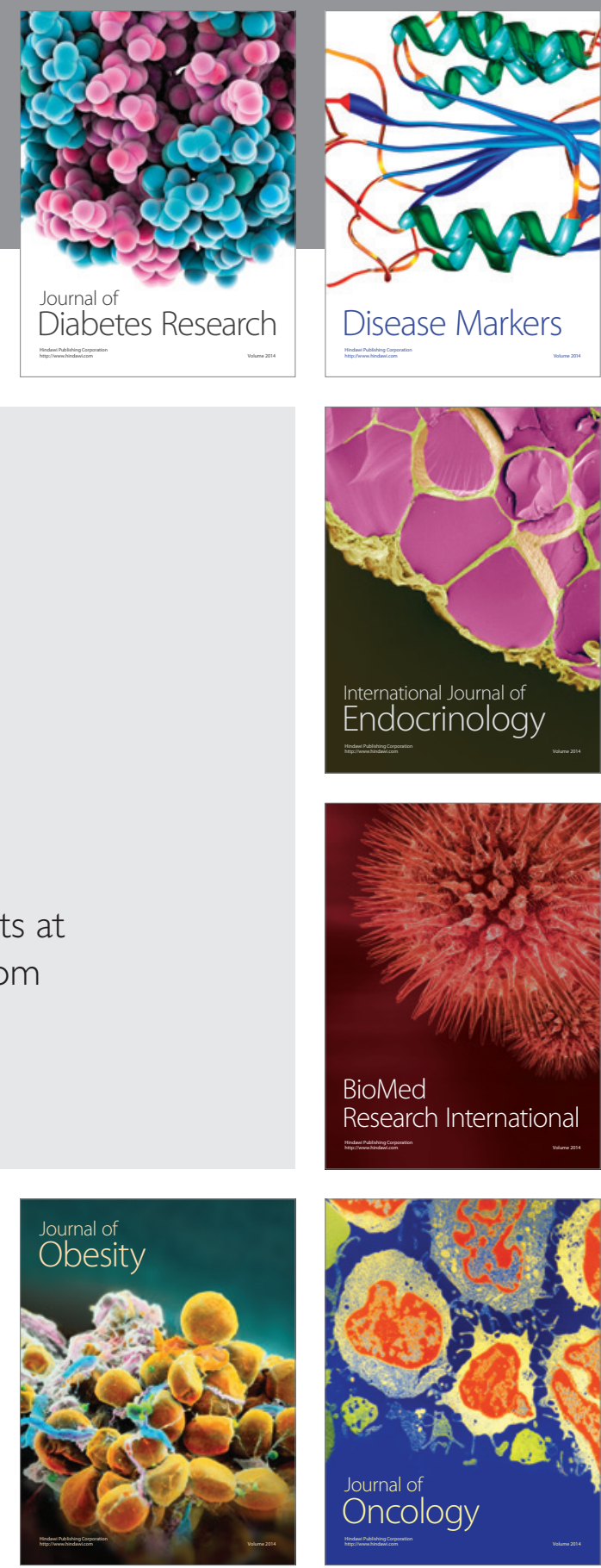

Disease Markers
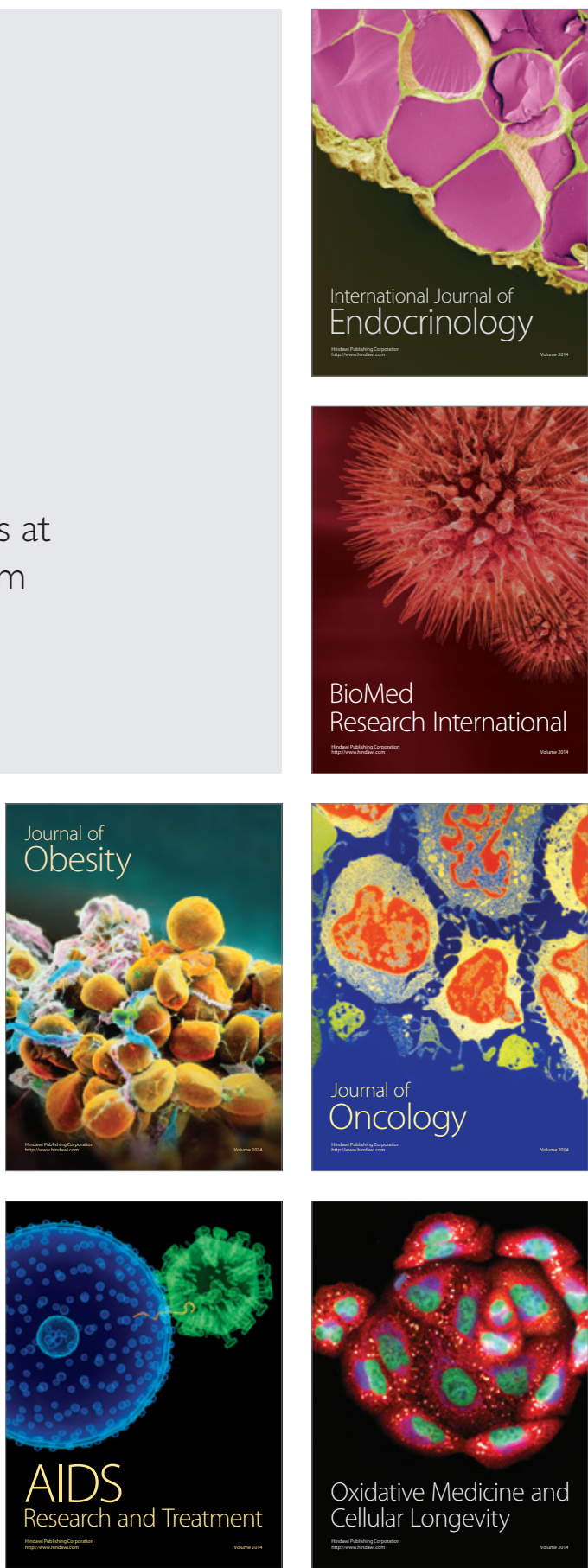